\title{
Unlocked evanescent waves in periodic structures
}

\author{
Muriel Botey, ${ }^{1, *}$ Yu-Chieh Cheng, ${ }^{2}$ Vicent Romero-Garcia, ${ }^{3}$ Ruben Picó, ${ }^{3}$ Ramon Herrero, ${ }^{2}$ \\ Victor Sánchez-Morcillo, ${ }^{3}$ and Kestutis Staliunas ${ }^{2,4}$ \\ ${ }^{1}$ Departament de Física i Enginyeria Nuclear, Universitat Politècnica de Catalunya, Urgell 187, Barcelona 08036, Spain \\ ${ }^{2}$ Departament de Física i Enginyeria Nuclear, Universitat Politècnica de Catalunya, Colom 11, Terrassa 08222, Spain \\ ${ }^{3}$ Instituto de Investigación para la Gestión Integrada de Zonas Costeras, Universitat Politècnica de València, \\ Paranimf 1, Gandia 46730, València, Spain \\ ${ }^{4}$ Institució Catalana de Recerca i Estudis Avançats (ICREA), Pg. Lluís Company 23, Barcelona 08010, Spain \\ ${ }^{*}$ Corresponding author: muriel.botey@upc.edu
}

Received February 26, 2013; accepted April 12, 2013;

posted April 29, 2013 (Doc. ID 185982); published May 23, 2013

\begin{abstract}
We predict the existence of evanescent modes with unlocked phases in two-dimensional (2D) dielectric periodic structures. Contrary to what is known for one-dimensional structures, where evanescent fields lock to the host modulation, we show that in 2D systems a new class of evanescent modes exists with an unlocked real part of the wave vector. Hence, beams constructed from such unlocked evanescent waves can exhibit spatial effects. A significant focalization of a beam propagating within the band gap of a flat photonic crystal slab is also shown. The predicted phenomenon is expected to be generic for spatially modulated materials. @ 2013 Optical Society of America

OCIS codes: (350.4238) Nanophotonics and photonic crystals; (260.5950) Self-focusing; (020.1670) Coherent optical effects.

http://dx.doi.org/10.1364/OL.38.001890
\end{abstract}

Periodic nanophotonic structures allow precise control over both temporal and spatial dispersion of light. Photonic crystals (PhCs) form band gaps (BGs), i.e., bands of frequencies at which plane waves do not propagate or, more precisely, propagate evanescently $[\underline{1}, 2]$. Recently, attention was paid to the possibility of engineering the propagation of light beams in such structures, mediated by anomalous spatial dispersion [3]. Among the reported novel effects are propagation without diffractive broadening (self-collimation) [4-8] , or negative diffraction (flat $\mathrm{PhC}$ lensing) [9-12]. Flat $\mathrm{PhC}$ lensing is accounted for by convex-curved isofrequency contours in wave vector, $k$, space $[13,14]$. The anomalous phase shifts accumulated trough a PhC slab are compensated by normal diffraction behind the structure, determining the focalization distance of the flat $\mathrm{PhC}$ lens. Beam focalization, however, has never been considered for frequencies within BGs.

Although evanescent waves decay exponentially, their transmission through finite-sized PhCs is never zero, and they can still lead to relevant effects [15]. In one-dimensional (1D) modulated structures, the phase of evanescent waves is fixed by the periodicity. The real part of their wave vectors locks to a multiple of the lattice vector of the host modulation, laying on straight lines in real $k$-space at the Brillouin zone (BZ) edges. The situation is generally analogous in two-dimensional (2D) PhCs. Note that neither beam focalization nor flat lens imaging is possible with locked waves.

We report here an unexpected observation: apart from the conventional locked evanescent waves, we find a new class of "unlocked" evanescent solutions. The wave vectors of such waves have a nonzero imaginary part, but its real part is not fixed by the host periodicity. Hence, they are able to present curved isofrequency contours and, potentially, "evanescent beams" constructed from them can show focalization behind a $\mathrm{PhC}$ slab, similar to flat PhC lensing for propagating modes [13]. We show that, under particular conditions, a substantial focalization can be obtained. Since all evanescent solutions are excluded from conventional $\omega(k)$ methods, such as the plane wave expansion (PWE), we apply an inverted or extended PWE (EPWE) method [16-18] or $k(\omega)$ method, which allows complex solutions, to determine the unlocked evanescent waves and to obtain the complex dispersion relations. We confirm the predicted beam spatial effects by a numerical integration based on the finite-difference time-domain (FDTD).

The various theoretical approaches to PhCs can be regarded either as an initial or a boundary condition problem. In the first case, the frequency of each spatial mode is determined for each given real-valued $k$; therefore, these methods are useful to describe infinite periodic structures. Frequency is purely real-valued in PhCs; only for gain/loss modulated materials can the imaginary part be nonzero, indicating the nonstationary growth or decay of propagating field [19]. The standard PWE is a modal method following this approach, widely used while limited to the description of propagating fields. On the other hand, to properly solve propagation through finite-sized PhCs, all field modes have to be considered, both propagating and evanescent. in a boundary condition problem where the amplitude, phase and frequency of the incident wave are fixed, and frequency is a realvalued magnitude. The exponential decay or growth of each mode in space is accounted for by the imaginary part of a complex-valued $k$, while its real part determines spatial dispersion [16-18,20]. In particular, the EPWE method considers the stationary Maxwell equations, fixes frequency and solves a non-Hermitian eigenvalue problem for $k$ [16-18].

Light propagation in 2D $\mathrm{PhCs}$, for certain directions and frequencies, can be approximately regarded as a 1D problem, if essentially only forward and backward waves come into play. This is the case for low frequencies in the crystallographic $\Gamma \mathrm{X}$ direction of a square lattice. Figure $\underline{1(\mathrm{a})}$ displays the real and imaginary parts 

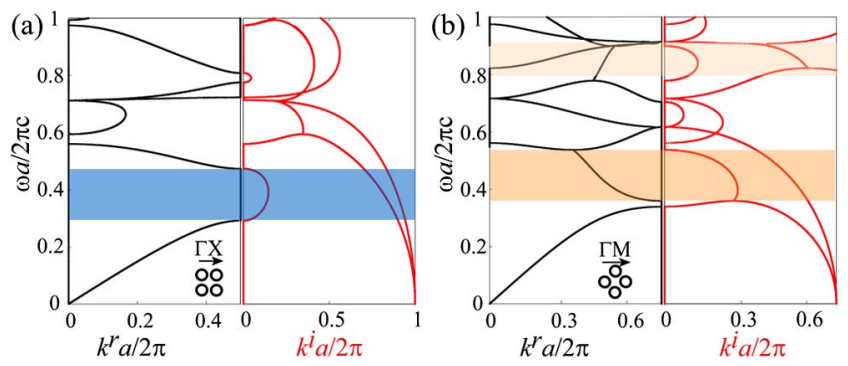

Fig. 1. TM complex dispersion relations calculated by the EPWE method for a high contrast $\mathrm{PhC}, \varepsilon_{1} / \varepsilon_{2}=11.34$, made of a square lattice of cylinders with radius $R=0.15 a$, where $a$ is the lattice constant; for propagation along (a) $\Gamma \mathrm{X}$ and (b) $\Gamma \mathrm{M}$. The real and imaginary parts of $k, k^{r}$ and $k^{i}$ (horizontal axis) are represented, depending on frequency, $\omega$ (vertical axis), in normalized units, where $c$ is the speed of light in a vacuum. The shaded areas show evanescent locked/unlocked modes.

of $k$, depending on frequency, as obtained by the EPWE method for TM polarization. As expected, the real part of $k$ locks to the boundary of the first $\mathrm{BZ}$ within the first $\mathrm{BG}$. However, for propagation along $\Gamma \mathrm{M}$ the situation is different [see Fig. 1(b)]. Within the first BG, the EPWE method yields the appearance of additional unlocked evanescent modes, where the real part of $k$ is not constant but depends on frequency. A careful inspection of Fig. 1(b) shows that at the lower part of the BG the evanescent mode remains locked, while increasing frequency the attenuation rates of two locked evanescent modes approach and merge, resulting in the appearance of an unlocked evanescent mode. Evanescent modes are represented by lines connecting the closest points of the dispersion curves from two adjacent propagation bands. If the frequency maxima and minima from both bands are not at high symmetry points of the BZ, the modes become unlocked, bearing some analogy with "indirect band gaps" known in semiconductors, e.g., [21]. The existence of such unlocked evanescent modes is the first basic result presented in this work. Moreover, analog solutions are also present in higher order BGs along the same $\Gamma \mathrm{M}$ direction as well as in the $\Gamma \mathrm{X}$ direction. We also report that for TE polarization unlocked evanescent modes appear, connecting the closest points of higher bands.

In the following, we concentrate on the diffractive propagation of monochromatic beams. Beam propagation is determined by isofrequency contours in $k$-space, shown in Fig. 2 for the first and second propagation bands. The top small frames, Figs. 2(a) and 2(d), show the real part of $k$, while the bottom frames, Figs. 2(b) and $2(\mathrm{e})$, depict the corresponding imaginary part, for the same frequency. We first consider a frequency lying already in the first $\mathrm{BG}$ for the $\Gamma \mathrm{X}$ direction, but still on the first propagation band for the $\Gamma \mathrm{M}$ direction [the inferior line on Fig. 2(c)]. Propagation is evanescent along $\Gamma \mathrm{X}$, the wave vectors are complex in this direction and their real parts lock to the boundary of the BZ, see Fig. 2(a). The flower-like pattern on Fig. 2(b) indicates the decay of evanescent modes along $\Gamma \mathrm{X}$. In $\Gamma \mathrm{M}$ direction $k$ 's are purely real-valued, and propagation is free or unlocked. Precisely this regime has previously been considered for self-collimation [8], or for PhC lensing [13], either due to flat or negatively curved segments in the

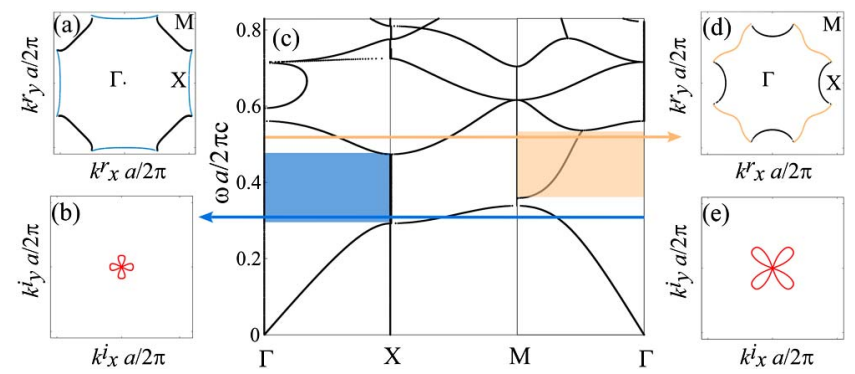

Fig. 2. TM band structure and complex dispersion relations calculated by the EPWE method, for the structure of Fig. 1 . Isofrequency contours at two frequencies; (a)/(b) and $(\mathrm{d}) /(\overline{\mathrm{e}})$ depict the real/imaginary parts of $k$. In (a)/(d) black (dark) lines correspond to the first propagation band, while blue/orange (light) lines correspond to the locked/unlocked evanescent modes. (c) Conventional representation of the band diagram, showing real parts of both evanescent and propagating bands.

spatial dispersion, respectively. The frames on the righthand side, Figs. 2(d) and 2(e), describe another situation. A constant frequency line at the top part of the BG in Fig. 2(c) intersects the second band along $\Gamma X$, but still lays in the BG along $\Gamma M$ direction. However, as shown in Fig. 2(d), the propagation is unlocked along both $\Gamma \mathrm{X}$ and $\Gamma \mathrm{M}$ directions. Comparing Figs. 2(b) and 2(e), the flower-like pattern in Fig. 2 (e) is now rotated, indicating evanescent propagation in the $\Gamma \mathrm{M}$ direction). Most importantly, the character of the evanescent wave is now completely different. The real part of $k$ is not locked to the edge of the $\mathrm{BZ}$, and the dispersion curve is no longer flat. In this particular case, as the frequency is close to the second band and hence laying in the second BZ, the curvature is slightly concave indicating normal diffraction. Note that the second $\mathrm{BZ}$ is adjacent to the depicted one translated by a reciprocal lattice vector. Hence, a convex segment of the spatial dispersion curve indicates negative diffraction, and may enable focalization of a beam by a thin PhC. However, differently from flat $\mathrm{PhC}$ lensing, we report here an analogous effect mediated by evanescent modes.

Next, we examine the predicted focalization of evanescent beams by direct numerical simulations. We generally find that strongly modulated PhCs with large index contrast and large filling factor result in concave spatial dispersion curves, leading to normal diffraction. Nevertheless, for low index contrasts, we obtain the opposite result: the curvature becomes positive along $Г M$ [see Fig. 3(a)]. As a consequence, focalization of an evanescent beam behind a thin PhC can be expected. Figure 3(b) shows the propagation of a linearly polarized Gaussian beam, obtained numerically by FDTD, where the intensity distribution clearly indicates focalization. Although the beam is relatively weak after evanescent propagation, the focalization behind the $\mathrm{PhC}$ is evident, especially when compared with a reference beam propagated in free space [see Fig. 3(b)]. Figures 3(c) and 3(d) summarize beam transmission in a frequency scan around the area of evanescent propagation. The intensity along the optical axis shows a maximum at a particular distance depending on frequency. Moreover, the $x$-position of the maximum (focusing distance) follows the tendency obtained from the calculation of isofrequency curves: increasing 

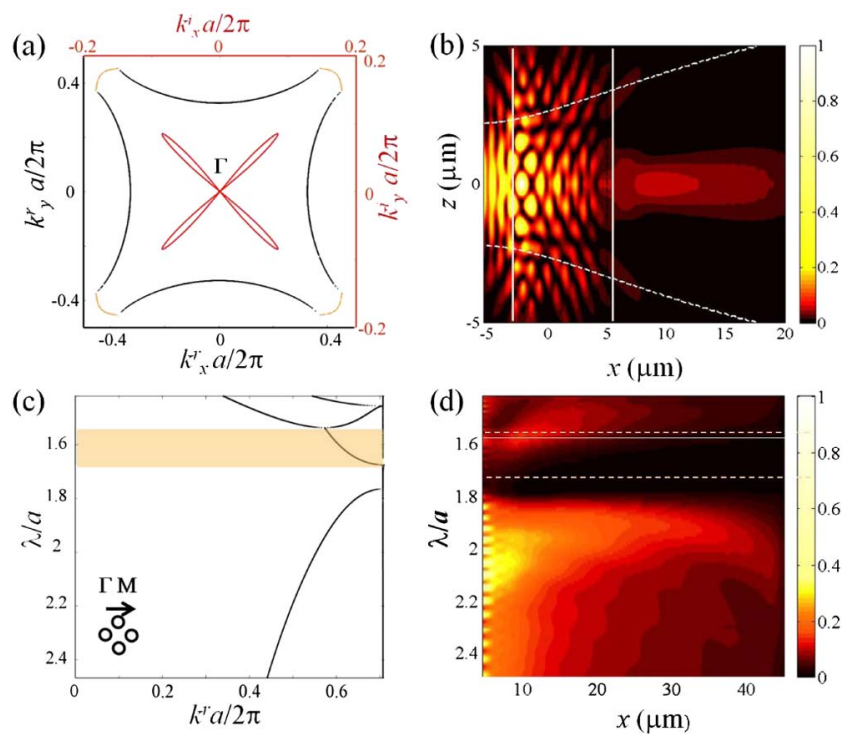

Fig. 3. Focalization of an evanescent beam by a low contrast PhC slab, $\varepsilon_{1} / \varepsilon_{2}=1.5$, made of a square lattice of cylinders, with $R=0.2 a$. (a) Isofrequency contours at $\omega a / 2 \pi c=0.633$ for complex $k$ 's. (b) Instantaneous field distribution inside and behind the system, for an incident Gaussian beam with the same central frequency, width $\omega_{0}=2 a$, propagating along $\Gamma \mathrm{M}$; the dotted line shows propagation in free space at $1 / \mathrm{e}^{2}$ maximum intensity. (c) ГM band diagram, whit the unlocked mode in the shaded area. (d) Transmitted intensity map along the propagation direction, $x$, on the beam axis $(z=0)$ for different normalized central wavelengths (vertical axis).

frequency, decreasing wavelength, curvature and focal distance increase. For completeness we also show the conventional focalization at the upper part of the first propagation band. Further increasing the crystal length the evanescent focusing is no longer observable.

In conclusion, we predict and numerically demonstrate unlocked evanescent waves in 2D PhCs. The common understanding is that evanescent waves in BGs lay at the edges of the BZ; i.e., that the field oscillations lock to the modulation of the host material. We show that, in addition, another class of unlocked evanescent modes exists. The arguments of the present work allow the expectation of them also in 3D. Unlocked evanescent waves are predicted by modifying the EPWE method, which extends the class of possible solutions including all evanescent modes. Besides, in order to prove their physical character, we show that beams formed by evanescent waves with unlocked wave vectors, hold spatial effects. Indeed, significant focalization of a Gaussian beam propagating within the $\mathrm{BG}$, in the unlocked evanescent regime through a low index contrast $\mathrm{PhC}$ slab, is numerically shown using direct FDTD simulations. For high contrast PhCs, we do not observe beam focalization in full accordance with our calculations. All calculations presented throughout the Letter are performed in TM polarization. Nevertheless, unlocked evanescent solutions are also found for the TE polarization. This suggests that the effect could be generic, originating from the periodicity and symmetry of the modulation rather than from the specific field and matter. Hence, we expect the predicted effect to be observed for the other kinds of waves in periodic structures, e.g., acoustic waves propagating in sonic crystals [22] or surface polariton waves, among others.

We acknowledge financial support by Spanish Ministerio de Ciencia e Innovación and European Union FEDER through project FIS2011-29731-C02-01 and -02. V. R. G. is grateful for the contract UPV CEI-01-11.

\section{References}

1. E. Yablonovitch, Phys. Rev. Lett. 58, 2059 (1987).

2. S. John, Phys. Rev. Lett. 58, 2486 (1987).

3. M. Notomi, Phys. Rev. B 62, 10696 (2000).

4. H. Kosaka, T. Kawashima, A. Tomita, M. Notomi, T. Tamamura, T. Sato, and S. Kawakami, Appl. Phys. Lett. 74, 1212 (1999).

5. D. Chigrin, S. Enoch, C. Sotomayor Torres, and G. Tayeb, Opt. Express 11, 1203 (2003).

6. R. R. Iliew, C. Etrich, U. Peschel, F. Lederer, M. Augustin, H. J. Fuchs, and A. Tunnermann, Appl. Phys. Lett. 85, 5854 (2004).

7. D. W. Prather, S. Shi, D. M. Pustai, C. Chen, S. Venkataraman, A. Sharkawy, G. J. Schneider, and J. Murakowski, Opt. Lett. 29, 50 (2004).

8. K. Staliunas and R. Herrero, Phys. Rev. E 73, 016601 (2006).

9. J. B. Pendry, Phys. Rev. Lett. 85, 3966 (2000).

10. P. V. Parimi, W. T. Lu, P. Vodo, and S. Sridhar, Nature 426 , 404 (2003).

11. P. Kockaert, P. Tassin, G. Van der Sande, I. Veretennicoff, and M. Tlidi, Phys. Rev. A 74, 033822 (2006).

12. P. Tassin, L. Gelens, J. Danckaert, I. Veretennicoff, G. V. der Sande, P. Kockaert, and M. Tlidi, Chaos 17, 037116 (2007).

13. C. Luo, S. G. Johnson, J. D. Joannopoulos, and J. B. Pendry, Phys. Rev. B 65, 201104 (2002).

14. E. Cubukcu, K. Aydin, E. Ozbay, S. Foteinopoulou, and C. M. Soukoulis, Nature 423, 604 (2003).

15. C. Luo, S. G. Johnson, J. D. Joannopoulos, and J. B. Pendry, Phys. Rev. B 68, 45115 (2003).

16. Y. C. Hsue and T. J. Yang, Phys. Rev. E 70, 016706 (2004).

17. V. Laude, Y. Achaoui, S. Benchabane, and A. Khelif, Phys. Rev. B 80, 092301 (2009).

18. V. Romero-García, J. V. Sánchez-Pérez, S. CastiñeiraIbáñez, and L. M. Garcia-Raffi, Appl. Phys. Lett. 96, 124102 (2010).

19. M. Botey, R. Herrero, and K. Staliunas, Phys. Rev. A 82, 013828 (2010).

20. C. S. Feng, L. M. Mei., L. Z. Cai, X. L. Yang, S. S. Wei, and P. J. Li, J. Phys. D 39, 4316 (2006).

21. C. Kittel, Introduction to Solid State Physics, 8th ed. (Wiley, 2005).

22. V. Romero-García, J. O. Vasseur, A. C. Hladky-Hennion, L. M. Garcia-Raffi, and J. V. Sánchez-Pérez, Phys. Rev. B 84, 212302 (2011). 